\title{
A STUDY OF VENTILATOR-ASSOCIATED PNEUMONIA, SURGICAL SITE INFECTION AND CENTRAL LINE-ASSOCIATED BLOODSTREAM INFECTION: INCIDENCE, OUTCOME, RISK FACTORS AND MEASURES TO BE TAKEN FOR PREVENTION IN A TERTIARY CARE TEACHING HOSPITAL
}

\author{
MABLE MARION*, LAIGY MARIAM MATHIAS, LEEMOL LALU, AKHILA S ARJUN \\ Department of Pharmacy Practice, Bapuji Pharmacy College, Davangere, Karnataka, India. Email: mable.m12@gmail.com
} Received: 25 May 2021, Revised and Accepted: 04 August 2021

ABSTRACT

Objective: Hospital-associated infections (HAIs) contribute to increased morbidity and mortality, excess healthcare cost, and prolonged hospital stay. This study aims to determine the incidence, risk factors of ventilator associated pneumonia (VAP), surgical site infection (SSI), and central line associated blood stream infection (CLABSI). Together it also assesses the knowledge and practice of nursing staff, physician in understanding HAIs and prevention, also to improve the core knowledge of administration procedures.

Methods: A prospective study was conducted among patients, nurses, and doctors of Shamanur Shivashankarapa Institute of Medical Sciences and Research Center hospital for a period of about 6 months. The data were collected, analyzed in terms of both inferential and descriptive statistics.

Results: The incidence rate of VAP was found to be $23.61 \%$. VAP was more prominent in males and occurred more in patients who received prolonged ventilation, supine position, and altered mental status, and these risk factors was found to be statistically significant (p<0.05). Acinetobacter was the commonly found causative organism for VAP. The mortality rate in our study was found to be 1 and was reported in early-onset VAP. SSI was more common in females and occurred mostly in elective surgeries with more comorbid conditions and American Society of Anesthesiologists score and these were found to be statistically significant $(\mathrm{p}<0.05)$. MRCONS and Escherichia coli were the most common pathogen identified. Mortality rate in our study was zero and 15 patients were clinically well. The incidence rate of CLABSI was found to be $31.25 \%$. Out of 32 centralized patients, only 10 developed CLABSI and is more prominent in males. Staphylococcus aureus was the common organism followed by E. coli. Mortality rate in our study was zero and all the patients became clinically well. From the questionnaire survey, all the doctors and nurses were aware of HAIs and the most common susceptible population according to them were inpatients and the most effective method to prevent HAIs is to wash hand properly, use gloves while doing procedures.

Conclusion: VAP was more prominent followed by SSI and CLABSI. Study clearly indicates that educational interventions should be implemented to address the gaps regarding knowledge and practice of nurse and physicians.

Keywords: Hospital associated infection, Ventilator-associated pneumonia, Central line-associated bloodstream infection, Discharge against medical advice, American Society of Anesthesiologists.

(C) 2021 The Authors. Published by Innovare Academic Sciences Pvt Ltd. This is an open access article under the CC BY license (http://creativecommons.org/ licenses/by/4.0/) DOI: http://dx.doi.org/10.22159/ajpcr.2021v14i9.42323. Journal homepage: https://innovareacademics.in/journals/index.php/ajpcr

\section{INTRODUCTION}

Healthcare-associated infection (HAI) is defined as a localized or systemic condition resulting from an adverse reaction to the presence of an infective agent or its toxins acquired from health care settings that were not incubating or symptomatic at the time of admission to the health facility [1]. HAIs include:

\section{Surgical site infection (SSI)}

SSIs are defined as infections occurring up to 30 days after surgery (or up to 1 year after surgery in patients receiving implants) and affecting either the incision or deep tissue at the operation site. Any purulent discharge, abscess, or spreading cellulitis at the surgical site during the month after the operation.

\section{Ventilator-associated pneumonia (VAP)}

VAP is defined as pneumonia occurring $48 \mathrm{~h}$ after patients are intubated and received mechanical ventilation (MV). Respiratory symptoms with a minimum of two of the subsequent signs and symptoms appearing during hospitalization: cough, purulent sputum, new infiltrate on chest radiograph in step with infection.
Centre line-associated bloodstream infection (CLABSI)

A CLABSI is defined as a laboratory-confirmed bloodstream infection not associated with an infection at another site that develops $48 \mathrm{~h}$ after central line placement. Patient with any of the subsequent signs and symptoms: fever $\left(>38^{\circ} \mathrm{C}\right)$, chills/rigors or hypotension, and a minimum of one positive blood culture not associated with contamination. HAIs have significant consequences on patients, their families, and therefore the community as a full. The foremost common consequences of HAIs are increased morbidity, mortality, and length of hospitalization. Such consequences contribute substantially to boost both the direct and indirect cost of the health care services, which leads to additional costs to treat infected cases. Hence, such a problem wastes the available resources which don't seem to be already enough, especially in developing countries. HAI is increasingly becoming a serious global public health problem-posing a good threat to patient safety. Hospitals are the potential source at the danger of acquiring an infection during health care delivery. HAIs contribute to increased attributable morbidity and mortality, excess health care cost and prolonged hospital stay. Data associated with the prevalence, risk factors, causative micro-organisms, and outcomes of infection are necessary to extend and maintain awareness of the impact of infection. Therefore, our study aims to critically review the incidence, risk 
factors, and outcome, and to conclude specific measures that ought to be undertaken to stop VAP, SSI, and CLABSI. This study also aims to spot gaps in nurses and doctors knowledge and practices regarding HAIs control measures to enhance these training courses and enhance future good practice.

\section{METHODS}

\section{Study design and population}

We conducted a prospective observational study among the 200 inpatients at Shamanur Shivashankarapa Institute of Medical Sciences and Research Center, Davangere, Karnataka for a period of 6 months. Ethical approval for the study was obtained from the Institutional Ethics Committee of Bapuji Pharmacy College, Davangere, Karnataka.

This study included patients admitted to OBG, Gen. Surgery, intensive care unit (ICU), Cardiology, Urology, Gen Medicine with assisted invasive $\mathrm{MV}$, inserted central venous line, and those who have undergone invasive surgery for $\geq 48 \mathrm{~h}$. This study excluded all the subjects with the presence of infections before being ventilated, before surgery, and before insertion of central line.

The collected data will be recorded in the HAI surveillance form by reviewing patient medication charts. The patient details will be obtained along with type of device used, and device days, HAI acquired after being ventilated, after surgery, and after the placement of central line, length of hospitalization, and relevant risk factors and comorbidities at the time of admission and daily monitoring. The patient will be followed up till they are discharged. The medical staffs will be provided with a questionnaire and the responded HAI questionnaire will be collected within 3-5 days and it will be analyzed to assess the knowledge of the medical staffs.

\section{Statistical analysis}

All the collected data were gathered within the sort of an excel spreadsheet with all protected health information that was removed before any statistical analysis. Data analysis was done through IBM SPSS Statistics, Version 23.0. Fisher's Exact test was accustomed to evaluate for the relationship within data. A p-value of $\leq 0.05$ was used to determine the statistical significance.

\section{RESULTS}

Among the 200 individuals enrolled in the study, 122 (61\%) were males and $78(39 \%)$ were females Table 1.

Most of the subjects belonged to the age group of 31-60 years (51.5\%). The mean age was found to be 68.15 Table 2 .

Out of 72 ventilated, 141 surgical, and 32 centrally catheterized patients, 17 developed VAP (40.4\%), 15 developed SSI (35.7\%), and 10 developed CLABSI (23.9\%) respectively Tables 3,4 and Fig. 1.

VAP was more prominent in males (70.5\%), SSI in females (53.3\%), and CLABSI in males (60\%). VAP, SSI, and CLABSI were prominent in the age group of $31-60$ Fig. 2 , which accounts for $70.5 \%, 60 \%$, and $40 \%$, respectively Fig. 3.

\section{Analysis of risk factors of VAP}

VAP occurred more in patients who received prolonged ventilation, supine position, and altered mental status. VAP occurred more Table 5 in patients who Table 6 received H2 receptor antagonists Table 7 and PPIs when compared to sucralfate. VAP was confirmed in patients who had CPIS score higher than 6 Fig 5.

\section{Analysis of risk factors for SSI}

The mean preoperative hospital stay of the patients was 2.63. All the patients received prophylactic antibiotics within $1 \mathrm{~h}$ before the Table 9 incision. American Society of Anesthesiologists (ASA) score of most of the patients was II (73.3\%) Table 10. SSI occurred mostly in Elective

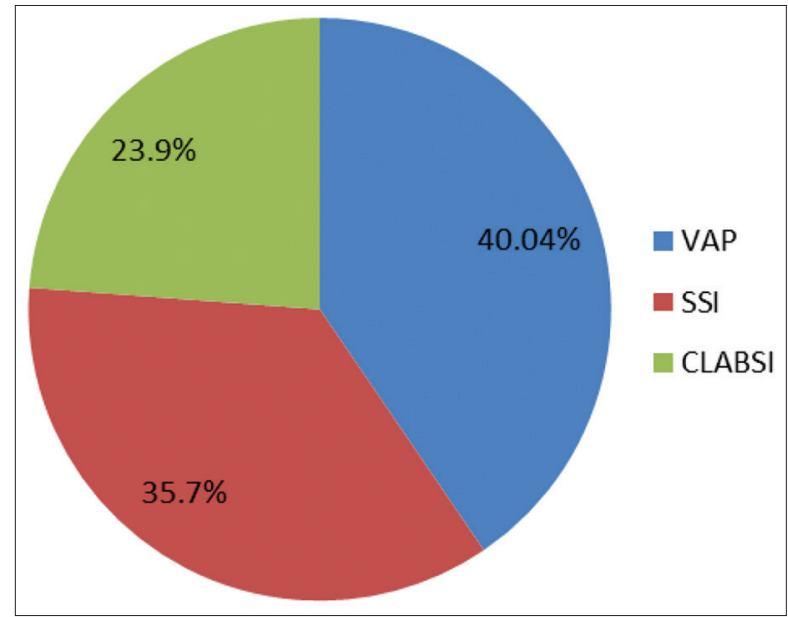

Fig. 1: Distribution of ventilator associated pneumonia, surgical site infection and central line associated blood stream infection

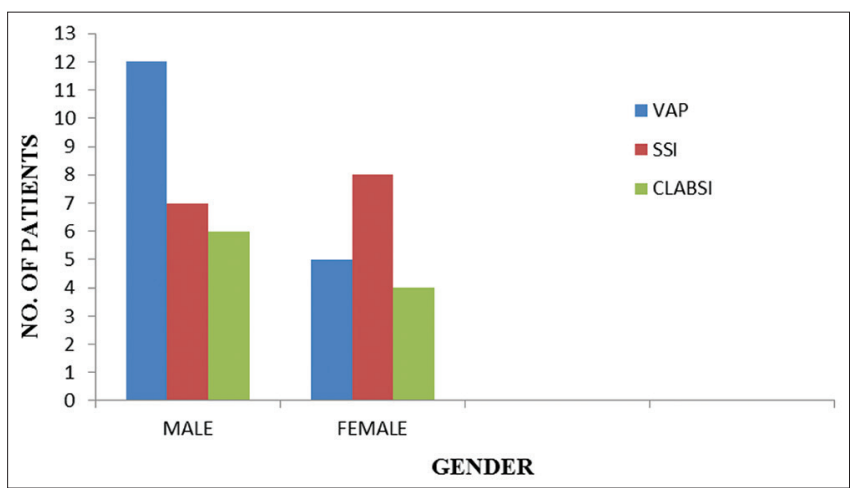

Fig. 2: Distribution of ventilator associated pneumonia, surgical site infection and central line associated blood stream infection based on gender

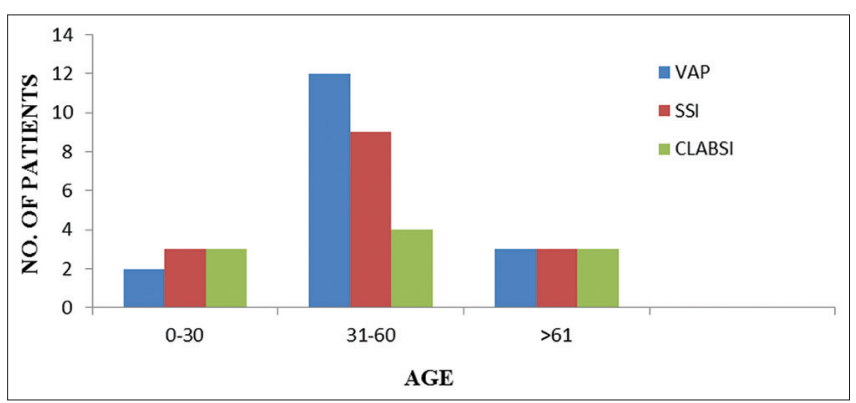

Fig. 3: Distribution of ventilator associated pneumonia, surgical site infection and central line associated blood stream infection based on age groups

surgeries because these patients presented Table 11 more comorbid illnesses. SSI emerged mostly in patients with clean-contaminated wounds Table 12-14 and Fig. 8.

\section{Analysis of Questionnaires}

25 questionnaires were distributed to 20 doctors and 5 nurses of critical care unit. 17 responded questionnaires were collected back from 12 doctors and 5 nurses.

\section{Knowledge section}

All of them were aware of the HAIs. Mostly isolated pathogens as per their knowledge were - E. coli, Klebsiella pnemoniae, Methicillinresistant Staphylococcus aureus (MRSA), Acinetobacter and S. aureus. 
Table 1: Distribution of Patients Based on Gender

\begin{tabular}{lll}
\hline Sex & No. of Patients & Percentage \\
\hline Male & 122 & 61 \\
Female & 78 & 39 \\
Total & 200 & 100 \\
\hline
\end{tabular}

Table 2: Distribution of Patients Based on Age

\begin{tabular}{lll}
\hline Age & No. of Patients & Percentage \\
\hline $0-30$ & 55 & 27.5 \\
$31-60$ & 103 & 51.5 \\
$>61$ & 42 & 21 \\
Total & 200 & 100 \\
\hline
\end{tabular}

Table 3: Incidence of VAP, SSI and CLABSI

\begin{tabular}{llll}
\hline HAIs & Population at Risk & No of cases & Incidence Rate (\%) \\
\hline VAP & 72 & 17 & 23.61 \\
SSI & 141 & 15 & 10.63 \\
CLABSI & 32 & 10 & 31.25 \\
\hline
\end{tabular}

VAP: Ventilator-associated pneumonia, SSI: Surgical site infection,

CLABSI: Central line-associated bloodstream infection, HAIs: Healthcare-

associated infection

Table 4: Distribution of VAP, SSI and CLABSI

\begin{tabular}{lll}
\hline HAIs & No. of Patients & Percentage \\
\hline VAP & 17 & 40.4 \\
SSI & 15 & 35.7 \\
CLABSI & 10 & 23.9 \\
Total & 42 & 100 \\
\hline
\end{tabular}

VAP: Ventilator-associated pneumonia, SSI: Surgical site infection,

CLABSI: Central line-associated bloodstream infection, HAIs: Healthcare-

associated infection

Table 5: Comparison of the Duration of MV and Occurrence of VAP

\begin{tabular}{lll}
\hline Days on MV & Cases & VAP \\
\hline$\leq 10$ & 60 & 8 \\
$>10$ & 12 & 9 \\
Total & 72 & 17 \\
\hline
\end{tabular}

MV: Mechanical ventilation, VAP: Ventilator associated pneumonia

Table 6: Occurrence of VAP in Supine and Semi Recumbent Position

\begin{tabular}{lll}
\hline Positions & Cases & VAP \\
\hline Supine & 33 & 13 \\
Semi recumbent & 39 & 4 \\
Total & 72 & 17 \\
\hline
\end{tabular}

VAP: Ventilator associated pneumonia

Table 7: Relation of VAP with Consciousness of the Patient

\begin{tabular}{lll}
\hline Status & Cases & VAP \\
\hline Conscious & 29 & 3 \\
Altered mental status & 43 & 14 \\
Total & 72 & 17 \\
\hline VAP. Ventilator associated pneumonia & &
\end{tabular}

Most common HAI seen according to their answers were SSI and bloodstream infections. The most susceptible population according to
Table 8: Outcome of Patients with VAP

\begin{tabular}{lll}
\hline Outcomes & Early-onset & Late-onset \\
\hline Expired & 1 & 0 \\
Clinically well & 4 & 10 \\
Increased hospital stay & 5 & 9 \\
DAMA & 1 & 1 \\
\hline DAMA: Discharge against medical advice, VAP. Ventilator-associated pneumonia
\end{tabular}

Table 9: Relation of Infection rate with Preoperative Hospital Stay

\begin{tabular}{lll}
\hline Pre. OP Stay (Days) & No. of Patients & No. of Infections \\
\hline $0-1$ & 47 & 2 \\
$2-6$ & 79 & 9 \\
$7-13$ & 15 & 4 \\
Total & 141 & 15 \\
\hline
\end{tabular}

Table 10: Determination of SSI by Surgical Wound Class

\begin{tabular}{lll}
\hline Class & No. of Patients & No. of Infections \\
\hline Clean & 79 & 5 \\
Clean Contaminated & 41 & 6 \\
Contaminated & 21 & 4 \\
Dirty & 0 & 0 \\
Total & 141 & 15 \\
\hline
\end{tabular}

SSI: Surgical site infection

Table 11: Determination of SSI by ASA Class

\begin{tabular}{lll}
\hline Class & No. of Patients & No. of Infections \\
\hline 1 & 54 & 1 \\
11 & 69 & 11 \\
111 & 18 & 3 \\
Total & 141 & 15 \\
\hline
\end{tabular}

SSI: Surgical site infection, ASA: American Society of Anesthesiologists

Table 12: Outcomes of Patients with SSI

\begin{tabular}{ll}
\hline Outcomes & No. of patients \\
\hline Expired & 0 \\
Clinically well & 15 \\
Increased hospital stay & 12 \\
DAMA & 0 \\
\hline DAMA: Discharge against medical advice, SSI: Surgical site infection
\end{tabular}

Table 13: CLABSI based on Number of Catheterized Days

\begin{tabular}{lll}
\hline Catheterized Days & No. of Patients & No. of Infections \\
\hline $0-9$ & 28 & 7 \\
$>9$ & 4 & 3 \\
\hline
\end{tabular}

CLABSI: Central line associated blood stream infection

Table 14: Outcome of Patients with CLABSI

\begin{tabular}{ll}
\hline Outcomes & No. of Patients \\
\hline Expired & 0 \\
Clinically well & 10 \\
Increased hosp stay & 4 \\
DAMA & 0 \\
\hline
\end{tabular}

DAMA: Discharge against medical advice, CLABSI: Central line associated blood stream infection

the doctors and nurses were inpatients. The most effective method to prevent HAI is to hand wash properly. 


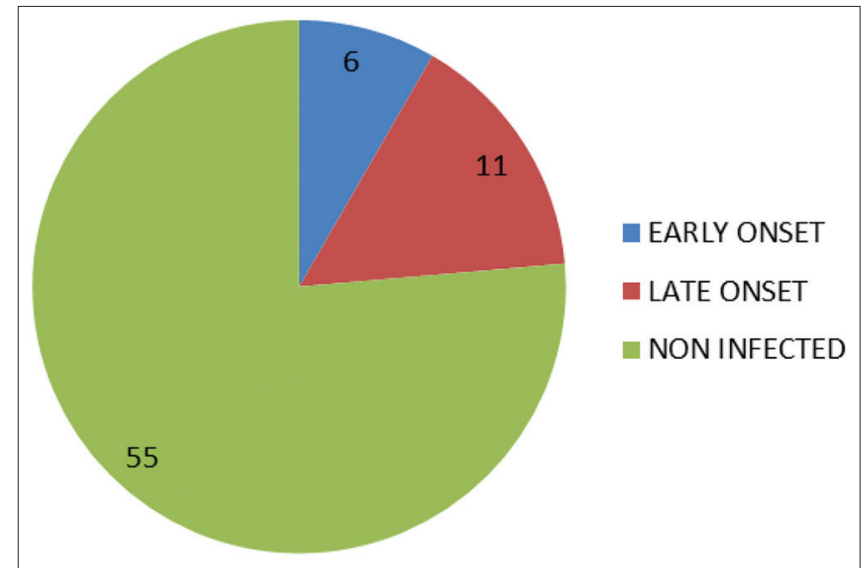

Fig. 4: Occurrence of ventilator associated pneumonia in ventilated patients

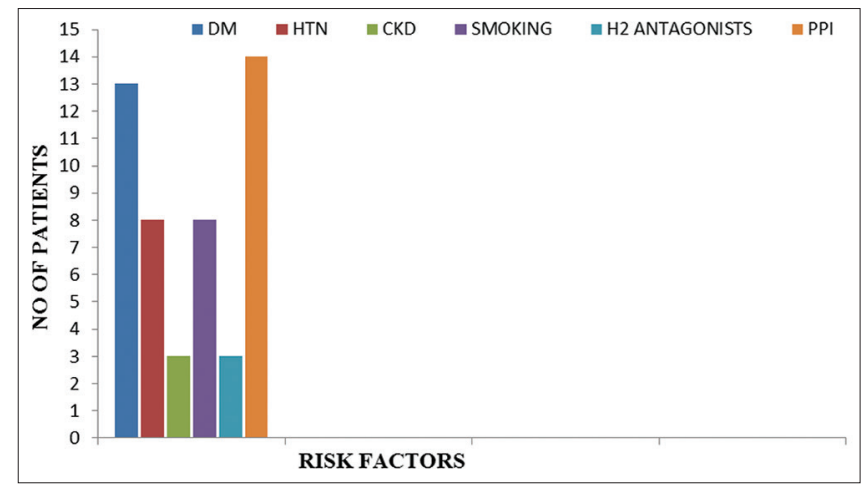

Fig. 5: Other possible risk factors of ventilator associated pneumonia were observed to be

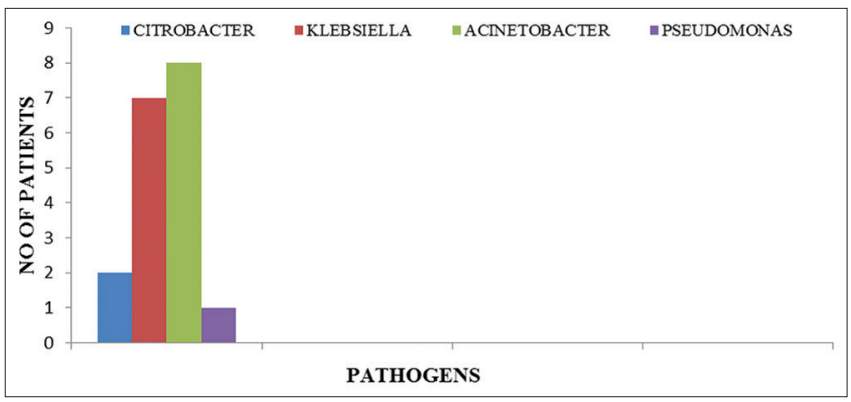

Fig. 6: Pathogens associated with ventilator associated pneumonia

\section{Practice section}

Doctors and nurses practiced the six step hand washing method before and after attending patients. They used medical utility gloves while performing physical examinations on patients.

\section{DISCUSSION}

In this study, the occurrence of VAP, SSI and CLABSI were more common among the age group of 31-60 years (51.5\%) [11,18]. The mean age was found to be 68.15. By assessing we have found that, out of 72 ventilated patients, 17 developed VAP (40.4\%), 141 patients who had undergone surgical procedure, 15 developed SSI (35.7\%) and out of 32 central lined patients, 10 developed CLABSI (23.9\%).

Incidence of VAP during this study was $23.61 \%$. VAP was more prominent in males (70.5\%). VAP occurred more in patients who received prolonged

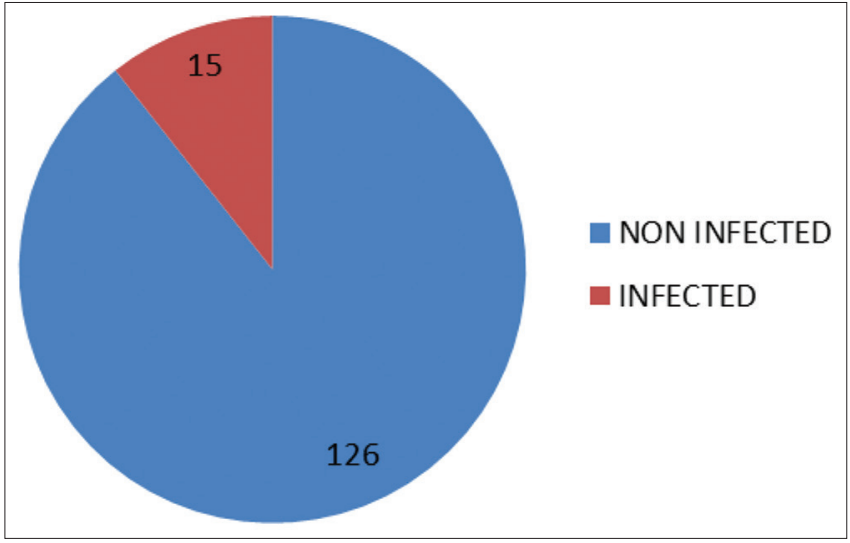

Fig. 7: Occurrence of surgical site infections in surgical patients

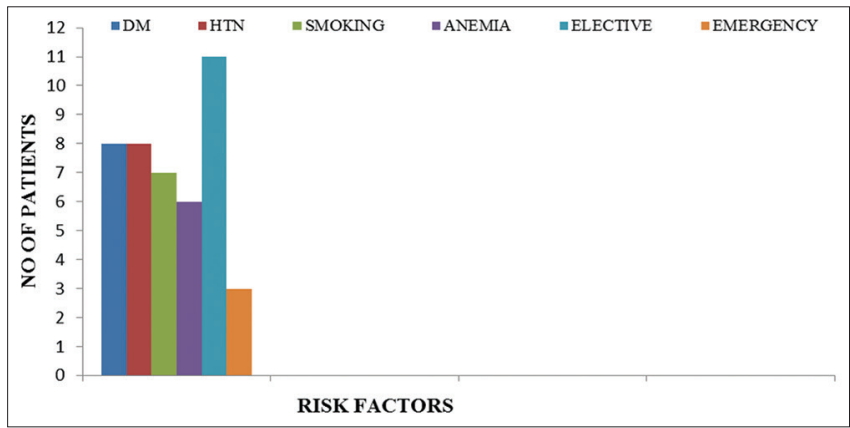

Fig. 8: Other possible risk factors associated with surgical site infections

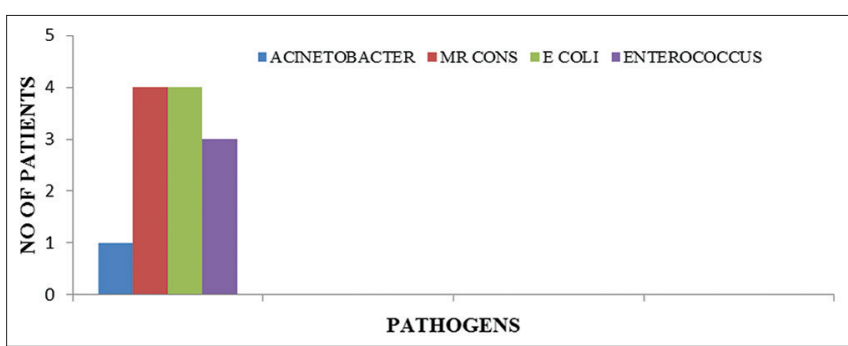

Fig. 9: Pathogens associated with surgical site infections

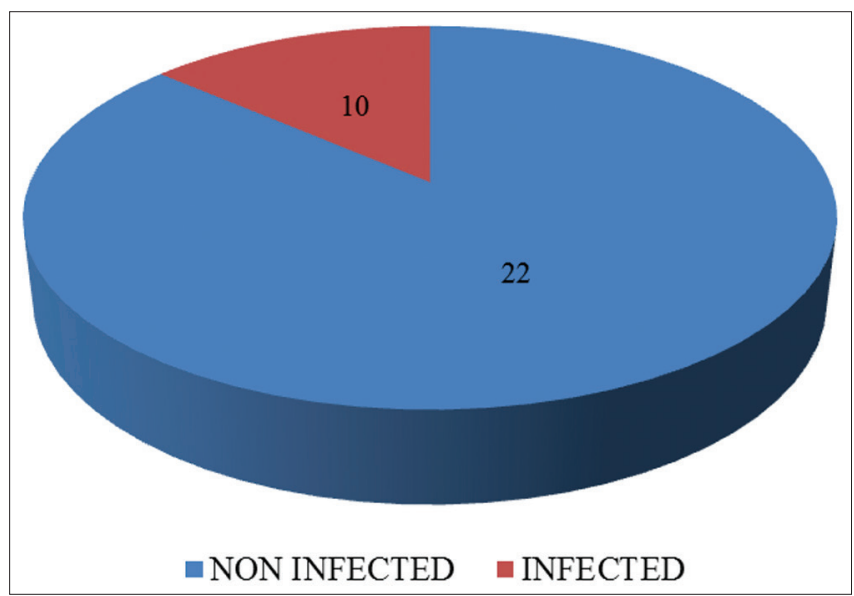

Fig. 10: Occurrence of central line associated bloodstream infections in central venous catheterized patients

ventilation, supine position and altered mental status. Re-intubation resulted in an exceedingly very high incidence of VAP and should flow 


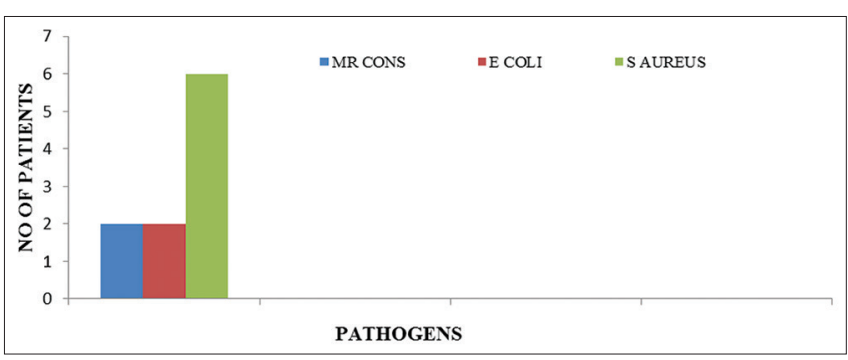

Fig. 11: Pathogens associated with central line associated bloodstream infections

from to impaired reflexes after prolonged intubation, increasing risk of aspiration. VAP occurred more in patients who received $\mathrm{H} 2$ receptor antagonist and PPIs in comparison to sucralfate. Sucralfate appears to possess alittle protective effect against VAP because it doesn't raise the gastric $\mathrm{pH}$ like $\mathrm{H} 2$ receptor antagonists. Therefore sucralfate should be used rather than $\mathrm{H} 2$ receptor antagonists [2,3]. VAP was confirmed in patients who had CPIS score on top of 6 . The observed possible risk factors are diabetes mellitus (DM), smoking, days on MV, mental status and patient position. the chance factors of VAP were found to be statistically significant $(\mathrm{p}<0.05)$.

The early onset VAP was 6 and late onset VAP was found to be 11 . The death rate during this study was found to be 1 and was reported in early onset VAP. Acinetobacter accounted for the very best number of cases followed by Klebsiella, Citrobacter, and Pseudomonas. Acinetobacter was the foremost commonest pathogen isolated in both early onset and late onset VAP [3].

Hand washing is well known as a very important measure to forestall VAP. per the 2004 Centre for Disease Control guidelines, hand should be washed before and after patient contact. Chlorhexidine is proven as effective in VAP. Oropharygeal care is another indicator to forestall VAP.

Incidence of SSI during this study was $10.6 \%$. SSI was more prominent in females $(53.3 \%)$ and it's been known that gender isn't a predeterminant risk of SSI $[9,13]$. The mean preoperative hospital stay of the patients was 2.63. Preoperative hospital stay for extended days expose the patient for contamination or colonization by the infectious pathogens which is able to increase the chance of SSI. All the patients received prophylactic antibiotics within $1 \mathrm{~h}$ before incision. ASA score of most of the patients were II (73.3\%). SSI occurred mostly in elective surgeries because these patients presented more co-morbid illnesses. SSI emerged mostly in patients with clean contaminated wound. Certain underlying conditions like DM, hypertension (HTN) and smoking significantly increase the danger of SSI. the danger factors of SSI were found to be statistically significant $(p<0.005)$. morbidity during this study was zero and 15 patients are clinically well [4].

The microbiological profile found among the patients who developed SSI was MRCONS and followed by Enterococcus and Acinetobacter. MRCONS and E. coli was the most pathogen identified and are liable for the event of SSI [13].

SSI is prevented by developing and following standardized order sets for every surgery to incorporate antibiotic name, timing of administration, weight based dose, re-dosing. Ensure pre-operative skin antisepsis and water shower. Develop and implement protocols to optimize glucose control all told surgical patients. Administer supplemental oxygen during the pre-operative, intra-operative and post-operative periods.

Incidence of CLABSI during this study was $31.25 \%$. Out of 32 central lined patients, only 10 patients developed CLABSI. CLABSI is more prominent in males $(60 \%)$. thanks to reduced number of incidence rate we couldn't specify the chance factors although possible risk factors could also be lack of aseptic technique, improper hand wash techniques. the danger factors of CLABSI weren't statistically significant. CLABSI occurred more in patients with 0-9 catheterized days. The microbiology of CLABSI during this study showed S. aureus as most typical organism cultured followed by E. coli and MRCONS. Death rate during this study was zero and every one the patients become clinically well [17].

Hand hygiene, maximal sterile barriers upon catheter insertion, use of chlorhexidine gluconate for skin preparation and avoidance of the vena for access site moreover as education can effectively control CLABSI rate. However CLABSI continues to be a threat to patient safety even within the prevention bundle era. Therefore, but insertion bundle strict maintenance bundles should be implemented.

To assess the knowledge 25 questionnaires were distributed to twenty physicians and 5 nurses and 17 responded questionnaires were collected back from 12 physicians and 5 nurses. All of them were attentive to the HAIs and therefore the commonest HAIs were SSI and bloodstream infection. Mostly isolated pathogens were - E. coli, K. pnemoniae, MRSA, Acinobacter and S. aureus and therefore the most susceptible population were inpatients. The foremost effective method to forestall HAI is handy wash properly.

\section{CONCLUSION}

HAI, are a reason for prolonged hospital stay, are inconvenient for the patient, constitute an economic burden on health care and increase the mortality of hospitalized patients.

VAP could be a major problem within the ICU resulting in longer hospital stay higher treatment costs and increased mortality and morbidity. Prolonged MV, patient position and mental status were the important risk factors. Additionally, prior use of antibiotics increases the chance of acquiring drug resistant pathogens. Effective medical aid and adequate staffing also impact on VAP prevention. Better knowledge of local patterns of pathogens causing VAP can help facilitate treatment choice.

SSIs remain a serious reason for morbidity and death among the operated patients and still represent a few fifth of all HAIs. Certain underlying conditions like DM, HTN, smoking and ASA score were statistically significant. Examining and identifying high risk patients and accordingly taking all appropriate care should be done to decrease the chance of SSIs. SSI will be prevented by developing and following standardized order sets for every surgical treatment to incorporate antibiotic name, timing of administration, weight based dose and redosing.

CLABSI is an infection which is best prevented instead of cured due to high associated mortality rates. Patients with CLABSIs have increased lengths of hospital stay, higher health care costs and attributable mortality. CLABSI is occurred more in patients with 0-9 catheterized days. Possible risk factors maybe lack of aseptic technique and improper hand wash techniques.

The study clearly indicates that the tutorial interventions are vital and may be implemented to deal with the gaps regarding knowledge and practice and to confirm that nurses use evidence-based prevention interventions.

\section{ACKNOWLEDGMENT}

We are also highly obliged to the subjects who gave us consent for submission of the manuscript.

\section{AUTHORS CONTRIBUTION}

Laigy Mariam Mathias and Leemol Lalu contributed in writing the manuscript whereas Mable Marion provided the particulars and details of the subjects. 


\section{CONFLICT OF INTEREST}

There is no conflict of interest between the authors.

\section{AUTHORS FUNDING}

None.

\section{REFERENCES}

1. Gosling R, Mbatia R, Savage A, Mulligan JA, Reyburn H. Prevalence of hospital-acquired infections in a tertiary referral hospital in northern Tanzania. Ann Trop Med Parasitol 2003;97:69-73.

2. Gadani H, Vyas A, Kar AK, A study of ventilator-associated pneumonia: Incidence, outcome, risk factors and measures to be taken for prevention. Indian J Anaesth 2010;54:535-40.

3. Ranjan N, Chaudhary U, Chaudhry D, Ranjan KP. Ventilator-associated pneumonia in a tertiary care intensive care unit: Analysis of incidence, risk factors and mortality. Indian J Crit Care Med 2014;18:200-4.

4. Leong G, Wilson J, Charlett A. Duration of operation as a risk factor for surgical site infection: Comparison of English and US data. J Hosp Infect 2006;63:255-62.

5. Carvalho RL, Campos CC, Franco LM, Ercole FF. Incidence and risk factors for surgical site infection in general surgeries. Rev Lat Am Enfermagem 2017;25:e2848.

6. Horan TC, Andrus M, Dudeck MA. CDC/NHSN surveillance definition of health care-associated infection and criteria for specific types of infections in the acute care setting. Am J Infect Control 2008;36:309-32.

7. Rello J, Ochagavia A, Sabanes E, Roque M, Mariscal D, Reynaga E, et al. Evaluation of outcome of intravenous catheterrelated infections in critically ill patients. Am J Respir Crit Care Med 2000;162:1027-30.

8. Iliyasu G, Dayyab FM, Habib ZG, Tiamiyu AB, Abubakar S, Mijinyawa MS, et al. Knowledge and practices of infection control among healthcare workers in a tertiary referral center in North-Western
Nigeria. Ann Afr Med 2016;15:34-40.

9. Iliyasu G, Dayyab FM, Habib ZG, Tiamiyu AB, Abubakar S, Mijinyawa MS, et al. Knowledge and practices of infection control among healthcare workers in a tertiary referral center in North-Western Nigeria. Ann Afr Med 2016;15:34-40.

10. Kirby JP and Mazuski JE. Prevention of surgical site infection SurgClin N, 2009; 89: 365-89.

11. Shah KH, Singh SP, Rathod J. Surgical site infections: Incidence, bacteriological profiles and risk factors in a tertiary care teaching hospital, Western India. Int J Med Sci Public Health 2017;6:173-6.

12. Amartej SS, BalijitSharanjit S, Iqbal S, Vaneet M. Prospective study of ventilator associated pneumonia incidence, risk factor, outcome and its prevention. J Anest Inten Care Med 2018;5:555666.

13. Leong G, Wilson J, Charlett A. Duration of operation as a risk factor for surgical site infection: Comparison of English and US data. J Hosp Infect 2006;63:255-62.

14. Wong ES. Surgical site infections.In:Mayhall CG, editor. Hospital epidemiology and infection control. $1^{\text {st }}$ ed.U.S.A: Williams and Wilkins; 1996.p.154-74.

15. Malhotra S, Sharma S, Hans C. Prevalence of hospital acquired infections in a tertiary care hospital in India. Int J Med Med Sci 2014;1:91-4.

16. Babbar P, Biswal M, Behera D, Gupta A. Healthcare associated infections in intensive care units: A pilot study in a Tertiary Care Public Hospital in India. J Prev Infect Control 2019;5:1-5.

17. Rode A, Bansod PY, Gujela A, Singh A. A study of central lineassociated bloodstream infections in intensive care unit: A prospective observational study. Int J Med Res Rev 2017;5:429-37.

18. Gastmeier P, Geffers C. Prevention of catheter-related bloodstream infections: Analysis of studies published between 2002 and 2005. J Hosp Infect 2006;64:326-35.

19. Drakulovic MB, Torres A, Bauer TT, Nicolas JM, Nogué S, Ferrer M. Supine body position as a risk factor for nosocomial pneumonia in mechanically ventilated patients: A randomized trial. Lancet 1999;354:1851-8. 